\title{
NOTE ON THE SINGULAR SOLUTIONS OF NONLINEAR ODES
}

\section{GABRIEL THOMAS}

Academie de Grenoble

Lycee des Portes de l'Oisans

Avenue Aristide Briand

38220 Vizille

France

e-mail: gabriel-an.thomas@ac-grenoble.fr

\begin{abstract}
Let an ODE $F\left(z, y, y^{\prime}\right)=0$, where $F$ is analytic in $\mathbb{C} \times \mathbb{C}^{n} \times \mathbb{C}^{n}$. The singular locus is the set $S=V\left(\operatorname{det} \frac{\partial F}{\partial y^{\prime}}\right)$. We are interested in the algebraic conditions for singular solutions to happen.

First we deal with the analytic case: the graph of a singular solution is embedded in $S$. We recall that such a solution may occur only when the differential ideal generated by $F\left(z, y, y^{\prime}\right)$ is not reducible. The existence of singular solutions is not generic and we show a relationship with the theory of differential-algebraic equations by Rabier and Rheinboldt [9]. This is also the adaptation to holomorphic differential equations of a result from Fukuda and Fukuda [2].

On the other hand, the Ritt-Raudenbusch theorems, related to the decomposition of differential ideals, give the algebraic conditions to get singular solutions for polynomial differential systems. This also indicates the non genericity of singular solutions existence.
\end{abstract}

2010 Mathematics Subject Classification: 34A09, $12 \mathrm{H} 05$.

Keywords and phrases: non-linear differential equations, singular solution, decomposition theorem.

Received April 25, 2016

(C) 2016 Scientific Advances Publishers 


\section{Introduction}

In classical differential algebra, we can read many papers on differential polynomials $([11,7,13])$. However, the pendulum equation

$$
\ddot{\theta}+\frac{g}{l} \sin \theta=0
$$

is not a polynomial in any sense. So the polynomial differential algebra does not allow to treat many equations that the physicists are naturally led to write. We will discuss the existence of singular solutions for systems of complex non-linear ODEs.

\section{Notations and Basic Results on Regular/Singular Solutions}

In this paper, the equation considered is a complex non-autonomous $n$-dimensional non-linear system of ODEs

$$
F\left(z, y, y^{\prime}\right)=0
$$

$n>0$ is an integer. $y^{\prime}=d y / d z$ is the first derivative of the vector-map $y=\left(y_{1}, y_{2}, \ldots, y_{n}\right)$. The map $F: \Omega \rightarrow \mathbb{C}^{n}$ is analytic over the open set $\Omega \subset \mathbb{C} \times \mathbb{C}^{n} \times \mathbb{C}^{n}$.

When we deal with geometric instead of differential objects, we may use the variable $p=\left(p_{1}, p_{2}, \ldots, p_{n}\right)$ for $y^{\prime}$.

Definition 1.1 (Jacobian). Let $\Phi: \mathbb{C}^{n} \rightarrow \mathbb{C}^{n}$ be a differentiable map of the complex variables $p_{1}, \ldots, p_{n}$. The Jacobian matrix of $\Phi$ is the square matrix

$$
M(\Phi)=\left(\left(\frac{\partial \Phi_{i}}{\partial p_{j}}\right)\right)_{1 \leq i, j \leq n},
$$

and we shall call the Jacobian of $\Phi$ the determinant

$$
\operatorname{Jac}(\Phi) \stackrel{\text { def }}{=} \operatorname{det} M(\Phi)
$$


For the Equation (2), we use the same notation:

$$
\operatorname{Jac}(F)(z, y, p)=\operatorname{det}\left(\left(\frac{\partial F_{i}}{\partial p_{j}}\right)\right)_{1 \leq i, j \leq n} .
$$

\subsection{General solution}

What the general solution of a non-linear ODE means has been discussed by several authors (see [11], [2] and more recently [5]). We recall this concept in the context of analytic differential equations:

Definition 1.2 (Regular point). Using the previous notations, a regular point of system (2) is any value $\left(z_{0}, y_{0}, p_{0}\right)$ such that $F$ is defined in an open neighbourhood of $\left(z_{0}, y_{0}, p_{0}\right)$ and that $\operatorname{Jac}(F)\left(z_{0}\right.$, $\left.y_{0}, p_{0}\right) \neq 0$.

Definition 1.3 (Solution). Any map $z \mapsto Y(z)$, holomorphic in a domain $^{1} U \subset \mathbb{C}$, is a solution of (2) whenever

$$
\forall z \in U, \quad F\left(z, Y(z), Y^{\prime}(z)\right)=0 .
$$

Consider a regular point $\left(z_{0}, y_{0}, p_{0}\right)$ of system (2): there is exactly one solution of the differential system (2) passing through $\left(z_{0}, y_{0}, p_{0}\right)$. It is sufficient to apply the implicit function theorem (to isolate $p$ ) in a neighbourhood of the regular point, then integrate the locally equivalent system using the standard Cauchy theory on explicit ODEs (see, e.g., Chapter 2 in Hille's book [4]).

Upon analytic continuation process, the unique solution defined in a neighbourhood of $z_{0}$ can be extended to a maximal open set, while still being solution of (2).

Definition 1.4 (Maximal solution). There is thus only one maximal solution at $\left(z_{0}, y_{0}, p_{0}\right)$ : the one defined in the maximal open set in $\mathbb{C}$, containing $z_{0}$ and satisfying

$$
\left(Y\left(z_{0}\right)=y_{0}, Y^{\prime}\left(z_{0}\right)=p_{0}\right) .
$$

\footnotetext{
${ }^{1} \mathrm{~A}$ domain is a non-empty open and connected set of $\mathbb{C}$.
} 
Definition 1.5 (Regular solution). At any regular point there is exactly one maximal solution. It is said to be a regular solution.

Definition 1.6 (General solution). The set of all the regular solutions of (2) define the general solution of (2).

\subsection{Singular solutions}

Definition 1.7 (Singular locus). The set of points $(z, y, p) \in \Omega$ such that

$$
\operatorname{Jac}(F)(z, y, p)=0
$$

is the singular locus of Equation (2). We note it $\mathcal{S}$.

Example 1.1. The 1-dimensional differential problem

$$
y^{\prime 2}=z+y^{2}
$$

has the singular locus $\{p=0\}$.

The 3-dimensional problem defined near $\left(z, y_{1}, y_{2}, y_{3}\right)=(0,0,0,1)$

$$
\left\{\begin{aligned}
y_{1}^{2} y_{1}^{\prime}+y_{1} y_{2}^{\prime 2}-y_{3} & =0 \\
y_{2}^{\prime} e^{-y_{2}+2 y_{3}}+2{y_{3}^{\prime}}^{2} & =z-y_{2} \\
2 \sqrt{y_{3}^{\prime 2}+1}-3 z y_{1}^{\prime} \sin \left(y_{2}-y_{1}\right) & =z \cos \left(z+y_{1}\right)
\end{aligned}\right.
$$

yields the Jacobian matrix

$$
M(z, y, p)=\left(\begin{array}{crc}
y_{1}^{2} & 2 y_{1} p_{2} & 0 \\
0 & e^{-y_{2}+2 y_{3}} & 4 p_{3} \\
-3 z \sin \left(y_{2}-y_{1}\right) & 0 & \frac{2 p_{3}}{\sqrt{p_{3}^{2}+1}}
\end{array}\right) .
$$


Its singular locus is defined by the analytic equation

$$
\operatorname{Jac}(z, y, p)=2 y_{1} p_{3}\left[\frac{y_{1} e^{-y_{2}+2 y_{3}}}{\sqrt{p_{3}^{2}+1}}-12 z p_{2} \sin \left(y_{2}-y_{1}\right)\right]=0 .
$$

The singular locus is either empty, or an analytic sub-variety of $\mathbb{C} \times \mathbb{C}^{n} \times \mathbb{C}^{n}$ defined as the zero set of a locally meromorphic map.

Definition 1.8 (Singular solution [2], p. 42). A singular solution $Y$ of Equation (2) is thus a differentiable map $z \mapsto Y(z)$ defined in an open set $U$ of $\mathbb{C}$ such that both following conditions hold:

$$
\forall z \in U, \quad F\left(z, Y(z), Y^{\prime}(z)\right)=0 \text { and } \operatorname{Jac}(F)\left(z, Y(z), Y^{\prime}(z)\right)=0 .
$$

By this definition, we see that the graph

$$
\left\{\left(z, Y(z), Y^{\prime}(z)\right) / z \in U\right\}
$$

of any singular solution is embedded in $\mathcal{S}$.

Example 1.2. Let us again mention the pendulum equation (1). Readily, we have the first integral

$$
F(\theta, \dot{\theta})=\frac{\dot{\theta}^{2}}{2}-\frac{g}{l} \cos \theta .
$$

The differential ideal $\{F\}$ is not irreducible: it contains by simple differentiation

$$
\dot{\theta}\left(\ddot{\theta}+\frac{g}{l} \sin \theta\right)=0
$$

Thus is it worth studying the singular solutions such that $\dot{\theta}=0$ ? Is there a chance to get an envelope $\theta=k$, where $k$ is a constant?

\subsection{Regular vs singular solutions}

We have the

Lemma 1.1. The general solution as defined in 1.6 does not contain any singular solution of (2). 
Proof. As the graph of a singular solution is embedded in $\mathcal{S}$, it does not contain any regular point.

Here a common question arises: it is well-known that, e.g., for some Clairaut equations (Fukuda [2], Example 1.4 (i), p. 44 ), there is a contact between a singular solution and a regular solution. Actually, a regular solution $Y(z)$ may contain some singular points, where $Y$ is still holomorphic. At least two differentiable solutions (one regular and one singular) get tangent at a precise point. Nevertheless

Lemma 1.2. Let $z \mapsto Y(z)$ be a regular solution, defined in a domain $U$ and

$$
\mathcal{G}=\left\{\left(z, Y(z), Y^{\prime}(z)\right) / z \in U\right\}
$$

its graph. The set $\mathcal{S} \bigcap \mathcal{G}$ is empty or made of isolated points.

Proof. Suppose that in $\mathcal{G}$, there is an accumulation of singular points near a point $\left(z^{*}, y^{*}, p^{*}\right) \in \mathcal{G}$. By continuity, this implies that in the neighbourhood of $\left(z^{*}, y^{*}, p^{*}\right)$, we have

$$
\operatorname{Jac}(F)(z, y, p)=0,
$$

so that $Y$ is a singular solution.

\section{Generic Differential Equations}

We recall in this part, that a generic differential equation cannot have any singular solution (see Thom [15]). The main reason is that the augmented system (4) is simply overdetermined.

\subsection{On singular solutions existence}

The genericity is the argument used in Fukuda's paper [2], in the context of smooth real differential equations. One of the main ideas is the use of the Malgrange preparation theorem to set the non-linear differential equation in a reduced form, in order to have locally (however 
generically) a discrete/finite covering: in the case of polynomials the solutions set has finitely many branches, above a regular point. The singular solutions are proved to be entirely embedded into the singular locus (Fukuda [2], Corollary 1.8): this is also the case considering analytic differential equations, whatever the base field (i.e., $\mathbb{R}$ or $\mathbb{C}$ ).

The generic impossibility of singular solution is clear following the way Rabier and Rheinboldt define the so-called index of differentialalgebraic equations (see [9]). The system (4) satisfied by a singular solution is made of $n+1$ equations:

$$
\left\{\begin{array}{l}
F\left(z, y, y^{\prime}\right)=0 \\
\operatorname{Jac}(F)\left(z, y, y^{\prime}\right)=0
\end{array}\right.
$$

Let us suppose the existence of a singular solution of graph $z \mapsto\left(z, y(z), y^{\prime}(z)\right)$, where $z$ belongs to a domain $U$. By elimination and eventually restricting $z$ to a non-empty open set $U_{1} \subset U$, in case Equations (4) are multivalued in $y^{\prime}$, at least one additional analytic equation can be obtained, which is independent on $y^{\prime}$ :

$$
G_{1}(z, y)=0
$$

defining a set $V_{1}=\left\{(z, y) \in U_{1} \times \mathbb{C}^{n} / G_{1}(z, y)=0\right\}$ : the system is a non-linear differential-algebraic equation (DAE). So the singular solution graph is embedded in the codimension 2 set $\mathcal{S} \bigcap V_{1}$. Supposing that the codimension value may be 1 only, is not generic: it would imply an algebraic condition relating $F, \operatorname{Jac}(F)$ and $G_{1}$. Following Rabier and Rheinboldt ([9], Theorem 5.1), we can define by induction a sequence $G_{2}, G_{3}, \ldots, G_{n+1}$ of analytic maps and a non-empty open set $U^{\prime} \subset U$, such that 


$$
\forall z \in U^{\prime}, \quad\left\{\begin{array}{l}
F\left(z, y(z), y^{\prime}(z)\right)=0, \\
\operatorname{Jac}(F)\left(z, y(z), y^{\prime}(z)\right)=0, \\
G_{i}(z, y(z))=0, i=1, \ldots, n+1 .
\end{array}\right.
$$

Thus generically we have $n+1$ analytic equation in $n+1$ unknowns: this yields an equation $g(z)=0$, where $g$ is a nonzero analytic map. No singular solution can exist by this last condition.

Corollary 2.1. The set of differential systems which do not contain any singular solution is dense among the analytic differential systems.

In the literature, the case of singular solutions is mentioned often to find these which are envelopes of the general solution $([4,1,6,14])$.

Corollary 2.2. Among the systems containing singular solutions, these such that singular solutions are envelopes of regular solutions are of codimension $\geq 1$.

The codimension being $\geq 1$ is the analytic condition formalizing the contact of order 1 when the general solution meets the singular locus (see [2]). In the other words, the set of differential systems with singular solutions not being envelopes are generic among these having singular solutions.

Now let us reformulate the results in an algebraic way.

\subsection{Algebraic conditions to have singular solutions}

In the case of differential polynomials, the theory developed mainly by Raudenbusch, Ritt and Kolchin applies: any system $F$ of such equations defines a radical differential ideal $\{F\}$. The ring of differential polynomials is Noetherian for radical ideals:

Theorem 2.1 (Ascending chain condition [10], p. 364). Any increasing sequence of radical differential ideals is stationnary.

As corollary any radical differential ideal is decomposable into prime ideals: 
Theorem 2.2 (Decomposition theorem for differential polynomials). Let $I$ be a radical differential ideal of polynomials: I is a finite intersection

$$
I=\bigcap_{j} \mathcal{P}_{j}
$$

where the $P_{j}$ are prime differential ideals (Raudenbusch [10], p. 365; Ritt [11], p. 42).

In the original Raudenbusch's paper $I$ is said to be a perfect differential ideal. Omitting the primes that contain the other, one gets the list of essential components which is unique upon eventual reordering. Now in the finite list $\mathcal{P}_{1}, \mathcal{P}_{2}, \ldots$, some components have their manifold of maximal dimension (see Ritt [11], p. 53-54): they form the general solution. All the other, if existing, contain the equations of the singular solutions. They are defined as the singular components and contain the Jacobian of the initial system.

As proved in Corollary 2.1, this last condition implies that polynomial differential systems containing a singular component cannot be generic. Unless a specific algebraic relation exists in the original equations, the condition that the Jacobian vanishes leads to the maximal ideal $\{1\}=\mathbb{C}\{z, y\}$ and to an empty set of solutions. Clearly, the ideal $\{1\}$ should not be taken into account when decomposing the ideal $I$. In the analytic case, we do not have such a nice and global decomposition, nor an easy definition of differential ideals associated to the differential system (2).

Suppose that $\mathcal{S}$ is non-empty and let $\left(z_{0}, y_{0}, p_{0}\right)$ be a point in $\mathcal{S}$. The concept of generic point, from the theory of schemes, should help to understand what an irreducible differential system means: an irreducible system contains one generic point only (see [3]). 
In $\mathbb{R}$ or $\mathbb{C}$, when an analytic differential system has singular solutions, it is made of a finite number of several irreducible components: a finite non-zero number of general systems of codimension 0 and a finite non-zero number of singular systems of codimension $\geq 1$. Any solution of a singular system is the solution of at least one general system.

\section{Personal Conclusion}

Are the singular solutions a myth? First they are non generic among the ordinary differential equations. Second point, when a system consists of the general solution and one or more singular components, these components are, roughly speaking, independent on the general component, since split by different prime ideals. The only case when a singular curve is the envelope of solutions of the general component may be seen as an algebraic coincidence or a curiosity. Does this really have any significant mathematical meaning?

\section{Acknowledgements}

The author is grateful to Dr. Romaric TYTGAT who brought inspiring support during this clarification work.

\section{References}

[1] M. Bhupal, On singular solutions of implicit second-order ordinary differential equations, Hokkaido Math. J. 32 (2003), 623-641.

[2] M. Fukuda and T. Fukuda, Singular solutions of ordinary differential equations, Yokohama Math. J. 15 (1977), 45-58.

[3] D. Eisenbud and J. Harris, Schemes: The Language of Modern Algebraic Geometry, Wadsworth \& Brooks/Cole Mathematics Series, 1992.

[4] E. Hille, Ordinary Differential Equations in the Complex Domain, Dover Publications Inc., 1976, Republished 1997.

[5] E. Hubert, Essential components of an algebraic differential equation, J. Symb. Comp. 28 (1999), 657-680.

[6] S. Izumiya and Y. Kurokawa, On systems of Clairaut type, Kodai Math. Journal 17 (1994), 636-643. 
[7] E. R. Kolchin, Some Problems in Differential Algebra, Proceedings Int. Congress of Mathematicians, Moscow 1966 (1968), 269-276.

[8] B. Malgrange, On nonlinear differential Galois theory, Chin. Ann. Math., bf 23B:2 (2002), 219-226.

[9] P. J. Rabier and W. C. Rheinboldt, A geometric treatment of implicit differentialalgebraic equations, J. of Diff. Equation 109 (1994), 110-146.

[10] H. W. Raudenbusch Jr., Ideal theory and algebraic differential equations, Trans. Am. Math. Soc. 36 (1934), 361-368.

[11] J. F. Ritt, Differential algebra, Amer. Math. Soc. Colloquium Publ. 33 (1948).

[12] J. F. Ritt, Algebraic aspects of the theory of differential equations, Amer. Math. Soc. Semi-centennial Publ. 2 (1938), 35-55.

[13] W. Sit, The Ritt-Kolchin's theory of differential poynomials, in Differential Algebra and related topics, World Scientific (2002), 1-70.

[14] C. N. Srinivasiengar, Singular solutions of simultaneaous ordinary differential equations, Proc. of the Indian Academy of Sciences - Section A 1(10) (1935), 668-693.

[15] R. Thom, Sur les équations différentielles multiformes et leurs integrals singulières, Boletim da Soc. Bras. De Mat., (1972), Colloque Elie Cartan, Paris 1971.

[16] H. Umemura, Galois theory and Painlevé equations, Séminaires et Congrès 14 (2006), 299-339. 